\title{
Coherent PONs for Next Generation Access: OIDMA versus OCDMA
}

\author{
Eslam A. El-Fiky and Ziad A. El-Sahn \\ Electrical Engineering Department \\ Faculty of Engineering \\ Alexandria University \\ Alexandria 21544, Egypt \\ Email: eslam_a_fiky@yahoo.com \\ Email: ziad.elsahn@ieee.org
}

\author{
Hossam M. H. Shalaby \\ Department of Electronics and \\ Communications Engineering \\ Egypt-Japan University of Science and Technology \\ Alexandria 21934, Egypt \\ Email: shalaby@ieee.org
}

\begin{abstract}
Coherent optical interleave-division multiple-access (OIDMA) has been recently proposed for next generation passive optical network (NG-PON) systems. In this paper we present a performance evaluation and comparison of coherent OIDMA and coherent optical code-division multiple-access (OCDMA) PONs in terms of ONU launch power, PON reach, bit error rate (BER), user bit rate, and number of users. Results reveal that OIDMA out-performs its counterpart in all aspects. Particularly, high user loading compared to OCDMA.
\end{abstract}

\section{INTRODUCTION}

In the last few years, bandwidth demand by end users increased dramatically owing to emerging new services such as high definition TV (HDTV), online gaming, and voice over IP (VOIP). Passive optical networks (PONs) have been accepted all over the world as the last mile broadband access technology that offers huge bandwidth, high user bit rate and long reach.

Current gigabit PONs and 10G-PONs employ time-division multiplexing (TDM) to share the bandwidth among different users by assigning different time slot to each optical network unit (ONU) [1]. Increasing bandwidth requirements pushes TDM-PONs to its capacity due to higher complexity. Next generation PONs (NG-PONs) are envisioned to support larger number of users, bandwidth, and distance coverage. Recently, different multiple access techniques have been proposed for NG-PONs e.g., wavelength-division multiplexing (WDM), optical code-division multiple-access (OCDMA), orthogonal frequecny-divison multiple-access (OFDMA), and interleaved frequecny-divison multiple-access (IFMDA), and optical interleave-division multiple-access (OIDMA). [2]-[7].

In this paper, we will focus on comparison between OCDMA and OIDMA systems. In multiuser systems, OCDMA suffers from multiple access interference (MAI). For large number of users, the excessive levels of MAI renders OCDMA not feasible due to the severe degradation of performance. Iterative turbo-type decoders have been studied in wireless communications area to mitigate MAI in CDMA systems [8], [9]. Progress in multiuser detection CDMA led to the so called IDMA [10], [11]. The main difference between CDMA and IDMA lies in the adopted method to distinguish between different users: CDMA employs user-specific spread- ing codes, whereas IDMA employs user specific interleavers as the only means of user separation.

In [7], performance of both non-coherent OIDMA and OCDMA is compared via simulations. Although the comparison itself is interesting, it is based on Matlab simulations where the simulation of some optical components was ignored, and many aspects were not considered: fiber length, fiber impairments, ONU launch power and user bit rate. Coherent OIDMA-PONs have been recently proposed for NGPONs [12], [13]. In this paper we present a comprehensive comparison of coherent OIDMA and coherent OCDMA for NG-PONs in terms of PON reach, number of ONUs, ONU launch power and bit error rate performance through a Matlab/Optisystem co-simulation. Results showed that coherent OIDMA outperforms its rival in all aspects. Coherent OIDMA can accommodate larger number of users, longer reach, and at relatively low launch power.

The rest of this paper is organized as follows. In Section II, the system model for OIDMA and OCDMA PONs is introduced and the receiver details are explained. Simulation results are presented and analyzed in Section III. Finally, the paper is concluded in section IV.

\section{SySTEM MODEL}

The system model for a $K$ user uplink architecture of coherent OIDMA PON is shown in Fig.1.

\section{A. Transmitter Side}

Information bits from the optical network unit (ONU) $k \in\{1,2, \ldots, K\}$, denoted by $\left\{b_{i}^{(k)} \in\{0,1\}, i=1, \ldots, N_{b}\right\}$ where $N_{b}$ is the information block length of user $k$, are spread using common low rate spreading codes of length $S$. This generates a coded sequence $\left\{c_{j}^{(k)}, j=1,2, \ldots, N_{c}\right\}$, where $N_{c}=N_{b} S$, then a chip level user specific interleaver is applied to produce the transmitted sequence $\left\{x_{j}^{(k)}, j=1,2, \ldots, N_{c}\right\}$. OIDMA uses user specific interleavers as the only means for user separation. The transmitted sequence modulates the optical carrier using quadrature phase shift keying (QPSK) modulation. Uplink signals coming from different users are combined at the remote node and passed to the feeder fiber 


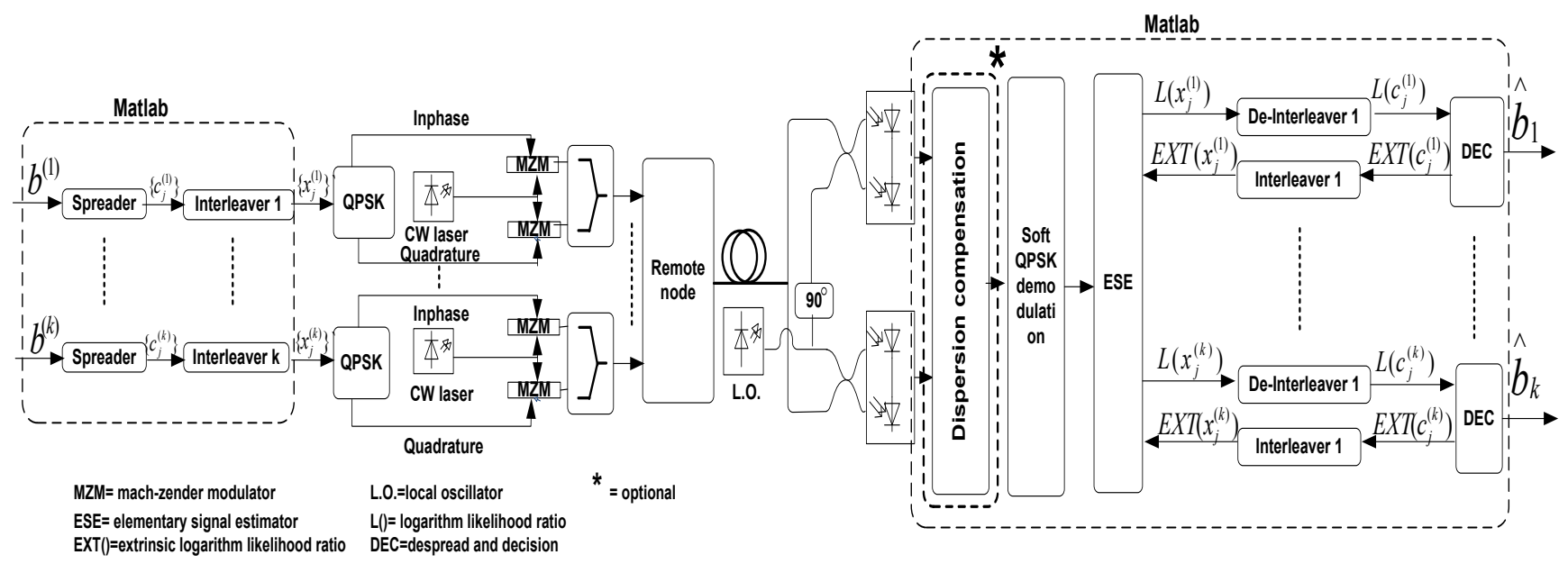

Fig. 1. Coherent OIDMA-PON uplink architecture.

to the optical line terminal (OLT). Removing the user specific interleavers $\pi^{(k)}$ and employing user specific spreading codes $\left\{s^{(k)} \in\{0,1\}, k=1,2, \ldots, K\right\}$ for user separation leads to OCDMA structure.

\section{B. Receiver Side}

The receiver front end is a coherent detection receiver. Electronic dispersion compensation may be applied to the output electrical signal before the soft QPSK demodulation. The output is then passed to the iterative chip-by-chip receiver.

\section{OIDMA Receiver}

OIDMA receiver comprises an elementary signal estimator (ESE) and a bank of $K$ single-user a posteriori probability decoders for the despreading operation (DEC) working in a turbo-type manner. In the wireless IDMA system, considering BPSK, the received symbols $r_{j}$ are given by [11], [14]

$$
r_{j}=\sum_{k=1}^{K} h^{(k)} x_{j}^{(k)}+n_{j}
$$

where $x_{j}^{(k)} \in\{+1,-1\}$ is the transmitted symbol from user $k$ at time instant $j, h^{(k)}$ is the channel coefficient for user $k$ assuming flat fading channel and $n_{j}$ is an additive white Gaussian noise (AWGN).

For the chip-by-chip decoding algorithm proposed in [11] we concentrate on each user's chip $x_{j}^{(k)}$ separately. Thus, (1) should be modified to

$$
r_{j}=h^{(k)} x_{j}^{(k)}+\zeta_{j}^{(k)}
$$

where $\zeta_{j}^{(k)}=r_{j}-h^{(k)} x_{j}^{(k)}$ represents the noise plus multiple access interference term with respect to $x_{j}^{(k)}$. From (1), the mean and variance of $r_{j}$ can be obtained as

$$
E\left(r_{j}\right)=\sum_{k=1}^{K} h^{(k)} E\left(x_{j}^{(k)}\right)
$$

and

$$
\operatorname{var}\left(r_{j}\right)=\sum_{k=1}^{K}\left|h^{(k)}\right|^{2} \operatorname{var}\left(x_{j}^{(k)}\right)+\sigma^{2}
$$

respectively. $E\left(x_{j}^{(k)}\right)$ and $\operatorname{var}\left(x_{j}^{(k)}\right)$ are initialized to be 0 and 1 , respectively, and then modified after each decoder iteration.

Using the central limit theorem for large number of users, $\zeta_{j}^{(k)}$ in (2) can be approximated by a Gaussian random variable with

$$
\begin{gathered}
E\left(\zeta_{j}^{(k)}\right)=E\left(r_{j}\right)-h^{(k)} E\left(x_{j}^{(k)}\right) \\
\operatorname{var}\left(\zeta_{j}^{(k)}\right)=\operatorname{var}\left(r_{j}\right)-\left|h^{(k)}\right|^{2} \operatorname{var}\left(x_{j}^{(k)}\right)
\end{gathered}
$$

The ESE outputs are the logarithm likelihood ratios (LLRs) of $x_{j}^{(k)}$ computed as

$$
\begin{aligned}
L\left(x_{j}^{(k)}\right) & =\log \frac{\operatorname{Pr}\left(x_{j}^{(k)}=+1 \mid r_{j}\right)}{\operatorname{Pr}\left(x_{j}^{(k)}=-1 \mid r_{j}\right)} \\
& =\log \frac{\exp \left(-\frac{\left(r_{j}-E\left(\zeta_{j}^{(k)}\right)-h^{(k)}\right)^{2}}{2 \operatorname{var}\left(\zeta_{j}^{(k)}\right)}\right)}{\exp \left(-\frac{\left(r_{j}-E\left(\zeta_{j}^{(k)}\right)+h^{(k)}\right)^{2}}{2 \operatorname{var}\left(\zeta_{j}^{(k)}\right)}\right)} \\
& =\frac{2 h^{k}\left(r_{j}-E\left(\zeta_{j}^{(k)}\right)\right)}{\operatorname{var}\left(\zeta_{j}^{(k)}\right)}, \forall k, j .
\end{aligned}
$$

For user- $k$, the corresponding ESE outputs $\left\{L\left(x_{j}^{(k)}\right), j=\right.$ $1,2, \ldots, J\}$ are de-interleaved to form $\left\{L\left(c_{j}^{(k)}\right), j=\right.$ $1,2, \ldots, J\}$ and delivered to the DEC for user- $k$. The DEC performs a soft-in/soft-out chip-by-chip de-spreading operation as follows [11]

$$
L\left(d_{1}^{(k)}\right)=\sum_{j=1}^{S} S_{j}^{(k)} L\left(c_{j}^{(k)}\right)
$$

where $\left\{S_{j}^{(k)}, j=1,2, \ldots, J\right\}$ is the spreading sequence of user $k$ such that $S_{j}^{(k)} \in\{1,-1\}$. The extrinsic LLR for a chip $c_{j}^{(k)}$ is given by 


$$
\operatorname{Ext}\left(c_{j}^{(k)}\right)=S_{j}^{(k)} L\left(d_{1}^{(k)}\right)-L\left(c_{j}^{(k)}\right)
$$

The extrinsic LLRs form the outputs of the DEC are fed back to the ESE after interleaving. In the next iteration, $\operatorname{Ext}\left(x_{j}^{(k)}\right)$ are used to update $E\left(x_{j}^{(k)}\right)$ and $\operatorname{Var}\left(x_{j}^{(k)}\right)$ as

$$
\begin{gathered}
E\left(x_{j}^{(k)}\right)=\tanh \left(\frac{\operatorname{Ext}\left(x_{j}^{(k)}\right)}{2}\right) \\
\operatorname{var}\left(x_{j}^{(k)}\right)=1-E\left(x_{j}^{(k)}\right)^{2}
\end{gathered}
$$

This iterative process is repeated for a predetermined number of iterations. After the final iteration, the data LLR $L\left(d_{1}^{(k)}\right)$ is hard limited to produce the estimate data sequence.

\section{OCDMA Receiver}

For fairness considerations, we use an iterative chip-by-chip decoder for the CDMA. An elementary signal estimator (ESE) block first calculates the LLRs of the received chips. The output LLRs are then correlated with the bipolar version of the spreading code [15] producing the despreaded LLR $L\left(y_{j}^{(k)}\right)$. The extrinsic LLR is then computed by

$$
\operatorname{Ext}\left(c_{j}^{(k)}\right)=L\left(y_{j}^{(k)}\right)-L\left(c_{j}^{(k)}\right)
$$

The extrinsic LLRs form the outputs of the DEC, and are fed back to the ESE after spreading again using the bipolar form of the spreading code. In the next iteration, $\operatorname{Ext}\left(x_{j}^{(k)}\right)$ are used to update $E\left(x_{j}^{(k)}\right)$ and $\operatorname{var}\left(x_{j}^{(k)}\right)$ in a manner similar to what is stated in OIDMA.

\section{Simulation Results}

In this section, we evaluate and compare the performance of coherent OIDMA-PONs and coherent OCDM-PONs via a Matlab/optisystem co-simulation.

\section{A. Simulation Setup}

The Simulation setup is shown in Fig. 1. The information block length is set to be 256 bits per user, and QPSK is used as a modulation scheme at the transmitter side. The laser power at the ONU is varied and the wavelength is set to $1550 \mathrm{~nm}$. We also consider a standard single mode fiber (SMF-28). All noise effects are considered at the receiver side. At the OLT, the local oscillator power is set to $0 \mathrm{dBm}$. The maximum chip rate is $10 \mathrm{Gchips} / \mathrm{s}$. The iterative decoder iterations are set to 10. For OCDMA-PON case we use Walsh-Hadamard codes as spreading codes, whereas we use alternating $\{1,0,1,0,1, \ldots\}$ spreading sequence for all users in OIDMA-PON case. We consider forward-error correcting (FEC) codes with $10^{-3}$ BER threshold. For Fig. 2 spreading codes of 64 chips length are utilized and number of users is set to 64 users. For Fig. 3 we vary the length of the spreading codes from 16 to 128 chips, either to accommodate more users (long spreading code) or to achieve higher bit rate (short spreading code), and PON reach is fixed at $100 \mathrm{~km}$. The ONU launch power is set to $0 \mathrm{dBm}$. Finally, for Fig. 4 we use spreading codes of length 64 for 100 and 110 users, and electronic dispersion compensation is added.

\section{B. Results}

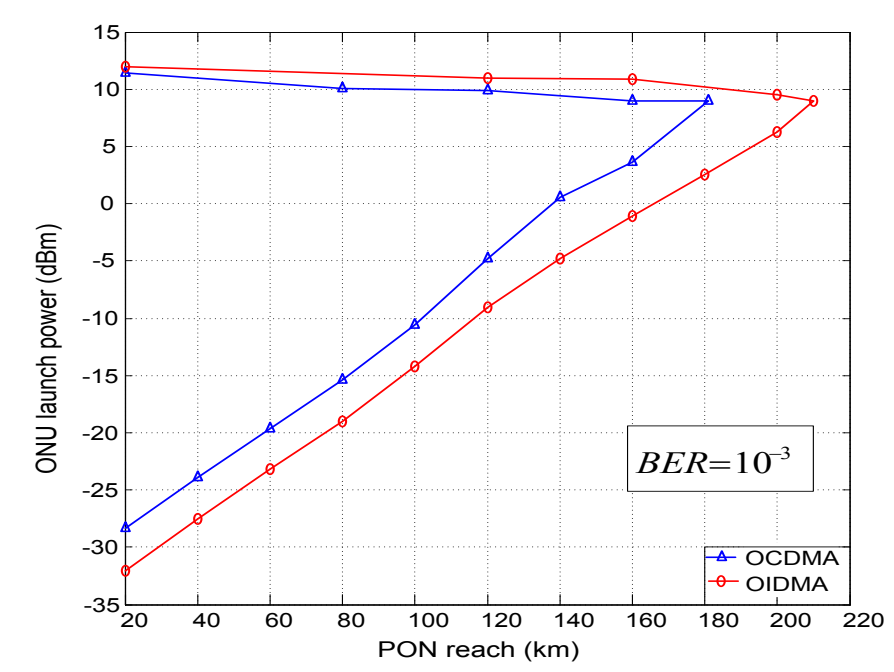

(a) ONU launch power versus PON reach for 64 users OCDMA and OIDMA PON systems.

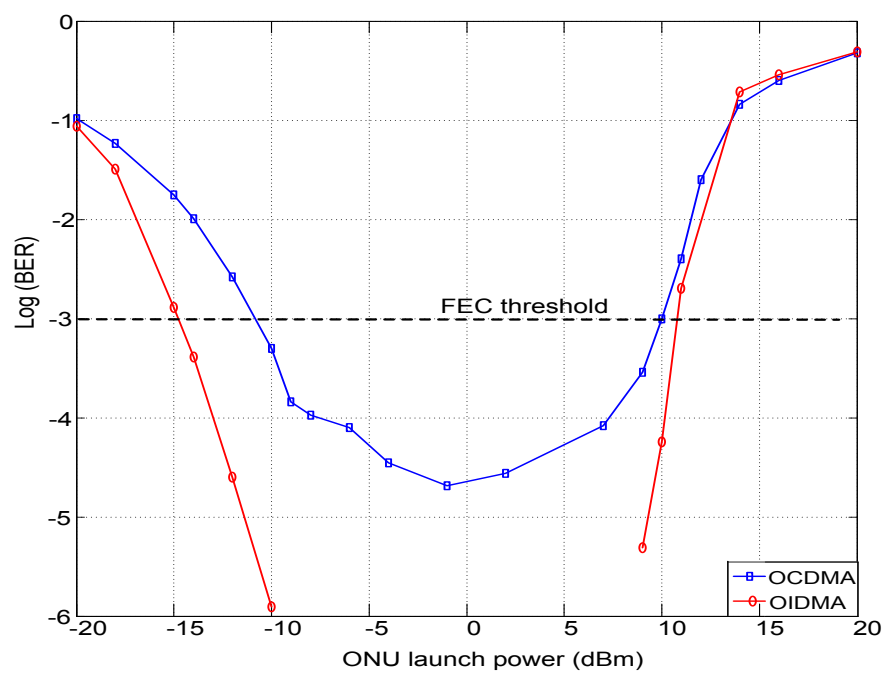

(b) BER performance of 64 ONUs and $100 \mathrm{~km}$ reach OCDMA and OIDMA PON systems versus ONU launch power.

Fig. 2. Uplink performance of coherent OCDMA and OIDMA PONs.

1) Effect of changing ONU launch power: Fig. 2.a shows the ONU launch power versus the PON reach for 64 users OIDMA and OCDMA PONs achieving the error free FEC threshold and Fig. 2.b shows BER performance versus ONU launch power for both systems PONs at $100 \mathrm{~km}$ reach. Both figures show the advantage of using OIDMA over OCDMA in PONs from both ONU launch power and PON reach perspectives. Several key observations can be observed from Fig. 2.a

- As expected, at relatively low launch power, the PON reach increases linearly as the launch power is increased. In such a linear regime the power simply compensates the fiber losses to achieve the same BER performance. 
At the same PON reach, the required ONU launch power to attain the FEC threshold for OIDMA is lower than for OCDMA. For example, at $100 \mathrm{~km}$ reach OCDMA requires $-10.6 \mathrm{dBm}$ launch power, whereas only $-14.2 \mathrm{dBm}$ for OIDMA is required .

- $\quad$ At the same ONU launch power, OIDMA can reach a significantly longer distance than OCDMA can. For example, at $0 \mathrm{dBm}$ launch power, OCDMA can support until $140 \mathrm{~km}$, but OIDMA can support until $170 \mathrm{~km}$; that is a $20 \%$ improvement in PON reach.

Figure 2.b shows that increasing power at same PON reach ameliorates the BER performance till reaching high power levels, where the non-linear effects of the fiber is not anymore negligible and the BER performance is degraded, so we have the U-shaped curve of Fig. 2.a.

2) Effect of changing length of spreading codes: Figure. 3 shows the ONU bit rate versus the maximum number of ONUs for both OIDMA and OCDMA systems at $100 \mathrm{~km}$ PON reach achieving the FEC threshold. It can be observed that a trade off exists between increasing ONU bit rate and increasing number of ONUs as shown in figure. The major advantage of OIDMA over OCDMA is that can accommodate significantly more users than the length of the spreading code and still achieving the FEC threshold. However, OCDMA is limited to the spreading code length or else the interference of the codes will severely degrade the performance. For example, at 312.5 Mbps (64 spreading length) OCDMA can accommodate only 64 users, whereas OIDMA can accommodate 101 users simultaneously for $100 \mathrm{~km}$ reach; that is an increase of about $58 \%$ in number of users at same user bit rate.

Figure. 4 shows the ONU launch power versus the PON reach for 100 and 110 users OIDMA system with and without electronic dispersion compensation for 64 spreading code length. It can be observed that OIDMA can also accommodate more than 101 users but for a PON reach less than 100 $\mathrm{km}$ at relatively low launch power. Also, adding dispersion compensation significantly enhances the PON reach and ONU launch power. For example, 100 ONUs OIDMA without dispersion compensation is limited to $108 \mathrm{~km}$ due to dispersion in fiber, but with dispersion compensation PON reach can be extended to $165 \mathrm{~km}$. That is an increase of about 53\% in PON reach.

\section{CONCLUSION}

Performance of coherent OIDMA and coherent OCDMA PONs in terms of ONU launch power, PON reach, user bit rate and number of ONUs has been presented. Results showed that OIDMA had a superior performance over OCDMA for NG-PONs in all aspects. OIDMA have a larger launch power range where BER is lower than error free FEC threshold. Also, OIDMA can accommodate a larger number of users simultaneously. In addition, PON reach is extended to reach more than $100 \mathrm{~km}$ for 100 users at a reasonably low launch power. Also, adding electronic dispersion compensation can even extend the PON reach further and decrease the launch power.

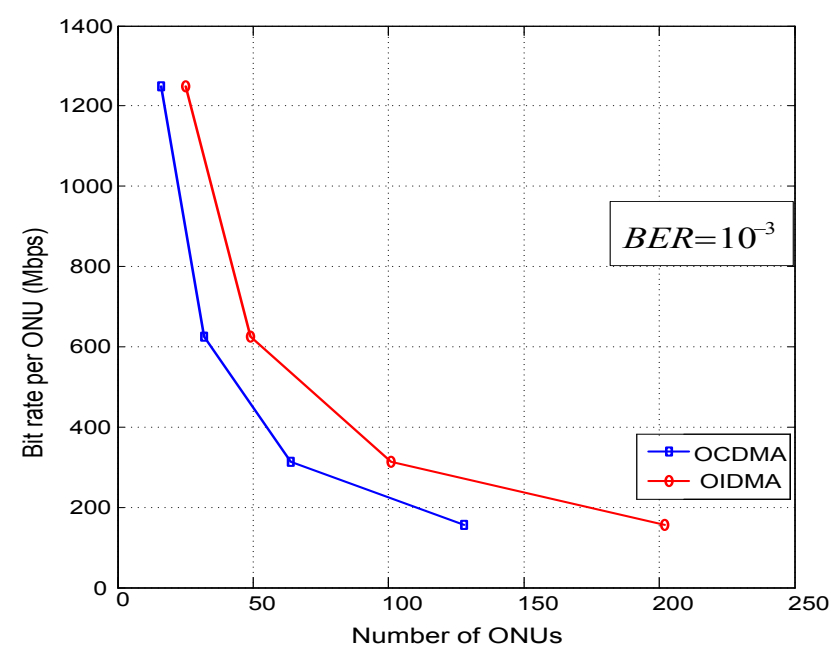

Fig. 3. User bit rate versus number of ONUs for OIDMA and OCDMA systems.

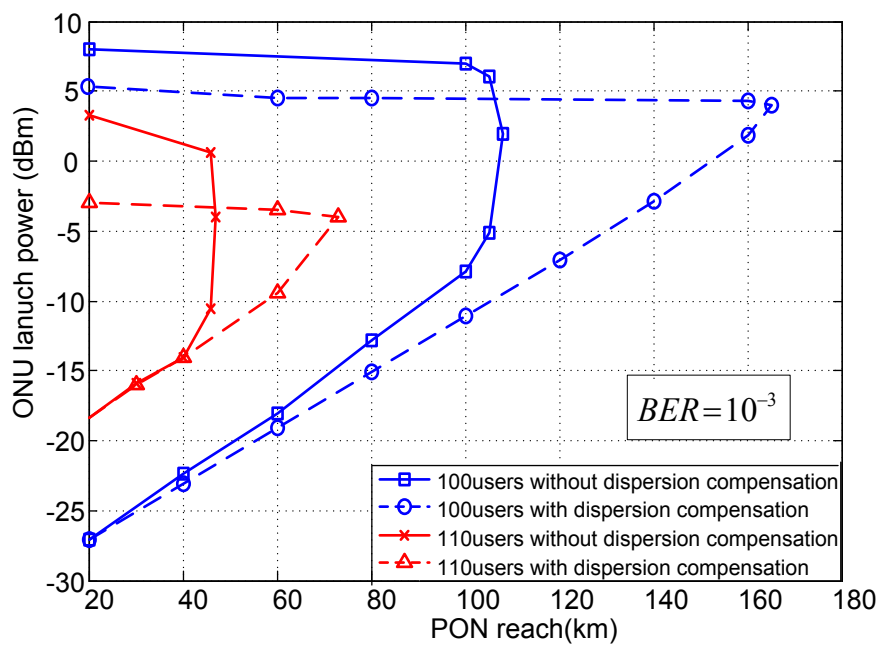

Fig. 4. ONU launch power versus PON reach for 100 and 110 users OIDMA system at $10 \mathrm{Gchip} / \mathrm{s}$ with and without dispersion compensation.

\section{REFERENCES}

[1] L. G. Kazovsky, W.-T. Shaw, D. Gutierrez, N. Cheng, and S.-W. Wong, "Next-generation optical access networks," Journal of Lightwave Technology, vol. 25, no. 11, pp. 3428-3442, 2007.

[2] R. Q. Shaddad, A. B. Mohammad, S. M. Idrus, A. M. Al-hetar, and N. A. Al-geelani, "Emerging optical broadband access networks from TDM PON to OFDM PON," $R N$, vol. 3, p. 4, 2012.

[3] J. A. Salehi, "Code division multiple-access techniques in optical fiber networks. i. fundamental principles," IEEE Transactions on Communications, vol. 37, no. 8, pp. 824-833, 1989.

[4] J. Armstrong, "OFDM for optical communications," Journal of lightwave technology, vol. 27, no. 3, pp. 189-204, 2009.

[5] N. Cvijetic, "OFDM for next-generation optical access networks," Journal of Lightwave Technology, vol. 30, no. 4, pp. 384-398, 2012.

[6] K.-i. Kitayama, Y. Yoshida, A. Maruta, K. Ishii, Y. Akiyama, M. Noda, K. Koguchi, M. Noami, K. Onohara, and T. Mizuochi, "Elastic and green optical access based upon coherent interleaved frequency division multiple access (IFDMA)," in Proc. Optical Fiber Communication Conference (OFC), 2013. 
[7] A. M. Morsy, E. A. El-Fiky, and H. M. Shalaby, "Performance analysis and comparison of optical IDMA and optical CDMA techniques using unipolar transmission scheme," in Proc. 18th European Conference on Networks and Optical Communications (NOC), 2013.

[8] R. H. Mahadevappa and J. G. Proakis, "Mitigating multiple access interference and intersymbol interference in uncoded CDMA systems with chip-level interleaving," IEEE Transactions on Wireless Communications, vol. 1, no. 4, pp. 781-792, 2002.

[9] X. Wang and H. V. Poor, "Iterative (turbo) soft interference cancellation and decoding for coded CDMA," IEEE Transactions on Communications, vol. 47, no. 7, pp. 1046-1061, 1999.

[10] L. Ping, L. Liu, K. Wu, and W. K. Leung, "Interleave division multipleaccess," IEEE Transactions on Wireless Communications, vol. 5, no. 4, pp. 938-947, 2006.

[11] W. K. Leung, L. Lihai, and P. Li, "Interleaving-based multiple access and iterative chip-by-chip multiuser detection," IEICE transactions on communications, vol. 86, no. 12, pp. 3634-3637, 2003.

[12] E. A. El-Fiky, A. M. Morsy, Z. A. El-Sahn, and H. M. Shalaby, "Coherent OIDMA technique for next generation long reach PONs," in Proc. CLEO, 2013.

[13] E. A. El-Fiky, Z. A. El-Sahn, and H. M. Shalaby, "Coherent long reach OIDMA-PONs enabled by electronic dispersion compensation," accepted at Asia Communications and Photonics Conference (ACP), 2013.

[14] L. Liu, W. Leung, and L. Ping, "Simple iterative chip-by-chip multiuser detection for CDMA systems," in Proc. Vehicular Technology Conference, 2003. VTC 2003-Spring., vol. 3, 2003, pp. 2157-2161.

[15] A. Tsuneda and T. Yoshida, "Performance evaluation of asynchronous DS/CDMA communications using unipolar codes," in Proc. 20th European Conference on Circuit Theory and Design (ECCTD), 2011, pp. 648-651. 